\title{
A Test Cassette for X-Ray-Exposure Experiments at the National Ignition Facility
}

K. B. Fournier, J. Celeste, V. Rekow, D. R. Bopp, M. J. May, J. H. Fisher, R. Horton, C. D. Newlander, P. Jenkins, K. Trautz

May 25, 2010

Review of Scientific Instruments 
This document was prepared as an account of work sponsored by an agency of the United States government. Neither the United States government nor Lawrence Livermore National Security, LLC, nor any of their employees makes any warranty, expressed or implied, or assumes any legal liability or responsibility for the accuracy, completeness, or usefulness of any information, apparatus, product, or process disclosed, or represents that its use would not infringe privately owned rights. Reference herein to any specific commercial product, process, or service by trade name, trademark, manufacturer, or otherwise does not necessarily constitute or imply its endorsement, recommendation, or favoring by the United States government or Lawrence Livermore National Security, LLC. The views and opinions of authors expressed herein do not necessarily state or reflect those of the United States government or Lawrence Livermore National Security, LLC, and shall not be used for advertising or product endorsement purposes. 


\section{A Test Cassette for X-Ray-Exposure Experiments at the National Ignition Facility}

K. B. Fournier, ${ }^{1}$ a) J. Celeste, ${ }^{1}$ V. Rekow, ${ }^{1}$ D. R. Bopp, ${ }^{1}$ M. J. May, ${ }^{1}$ J. H. Fisher, ${ }^{2}$, b) R. Horton, ${ }^{2}$ C. D. Newlander, ${ }^{2, c)}$ P. Jenkins, ${ }^{3,}$ ) and K. Trautz ${ }^{3}$

${ }^{1)}$ Lawrence Livermore National Laboratory, Livermore, California 94551, USA

2) Gray Research, Inc., 655 Discovery Drive, Suite 300, Huntsville, AL 35806

${ }^{3)}$ Naval Research Laboratory, Washington, DC 20375

(Dated: 25 May 2010)

We present the design and operation of a test cassette for exposure of samples to radiation environments at the National Ignition Facility. The samples had normal lines of sight to the x-ray source in order to have uniform x-ray illumination. The incident x-radiation onto the samples was determined by the choice of filter thicknesses and materials. The samples were held at precise locations in the target chamber in order to have a known fluence incident. In the cassette, the samples were held in place in such a way that a minimal 'line contact' allows them to have the maximal mechanical response to the x-ray load. We present post-shot images of the debris found on films used for filters, and pre- and post-exposure specimens.

\section{INTRODUCTION}

Recent experiments at the National Ignition Facility $(\mathrm{NIF})^{1,2}$ have demonstrated NIF's ability to produce with high precision a reproducible, high-flux x-ray environment ${ }^{3}$. Such environments are essential for studying $\mathrm{x}$-ray interactions with matter ${ }^{4-6}$. In this paper, we present the requirements that led to the design and operation of the X-Ray Source Application (XRSA) test cassette that was used for sample-exposure experiments at NIF. Such studies are relevant to looking at radiation damage to materials, radiation ablation off surfaces, and the effects of radiative heating and cooling on test particles. The need to introduce samples to the target chamber and recover them post shot is not limited to studying $\mathrm{x}$-ray interactions with matter; as an ignition platform becomes a reality, samples of activated materials will need to be recovered as radiochemical diagnostics of fusion yield ${ }^{7}$. Our test cassette can also be applied to collecting solid debris from target shots at NIF in order to characterize the NIF chamber's debris environment. Our design is particularly useful when it is important for the normal to the surface of the collection plate or the specimen to be exposed on a radial line of sight to the $\mathrm{x}$-ray source.

\section{REQUIREMENTS AND DESIGN CONSIDERATIONS}

The NIF target chamber is $10 \mathrm{~m}$ in diameter, with numerous ports in the chamber wall for quads of laser beams to enter as well as ports at many locations for fixed (quasi-permanent) diagnostic installations ${ }^{8}$. In ad-

\footnotetext{
a) fournier2@llnl.gov

b) jfisher@gray-research.com

c) cdavidnewlander@yahoo.com

d)Phillip.Jenkins@nrl.navy.mil
}

dition, there are three diagnostic instrument manipulators (DIMs) ${ }^{9}$ at set locations on the target chamber (two on the chamber's equator, one at the chamber's upper pole) that allow for servicing or change of a diagnostic during a shot cycle.

For our XRSA program, we desire to turn around shots as quickly as possible and we need to be able to service the sample cassette during a NIF shot cycle. We accomplish this by being able to install and remove our test cassettes through the access ports on the side of the DIMs, see Fig. 1. Further, the interface between the DIM and the NIF target chamber is a 30 -cm-diameter gate valve that is opened after DIM operations are completed and the vacuum in the DIM is pumped to a level compatible with the target chamber vacuum $\left(\approx\right.$ few $\times 10^{-5}$ Torr $)$. Further, we desire to maximize the number of test specimens fielded on each shot. For these first experiments, we knew that our test samples would be circular with diameters no larger than $38.1 \mathrm{~mm}$ (1.5"). Given the spaceenvelope constraints, we were able to optimize the testcassette design in such a way as to test eight samples per shot.

\section{A. Sample Holders and Sample Contact}

Our first test series had numerous requirements that define how specimens are to be held and oriented. We had to accommodate specimen exposure areas up to $25.4 \mathrm{~mm}$ in length or diameter, which is the size of the aperture for round samples in the sample holder, or "paddle", shown in Fig. 2. Dimensions of key parts of the XRSA cassettes are tabulated in Table I. All the components of the XRSA cassette (except for the rotation shoulders and filter films, which are discussed below), as well as the rails to which the cassette attaches are made of aluminum 6061-T6 alloy. The fasteners that hold the components together are made of stainless steel. The components of the sample holder in Fig. 2 include the paddle that has the receptacle for the sample (green piece in the mid- 

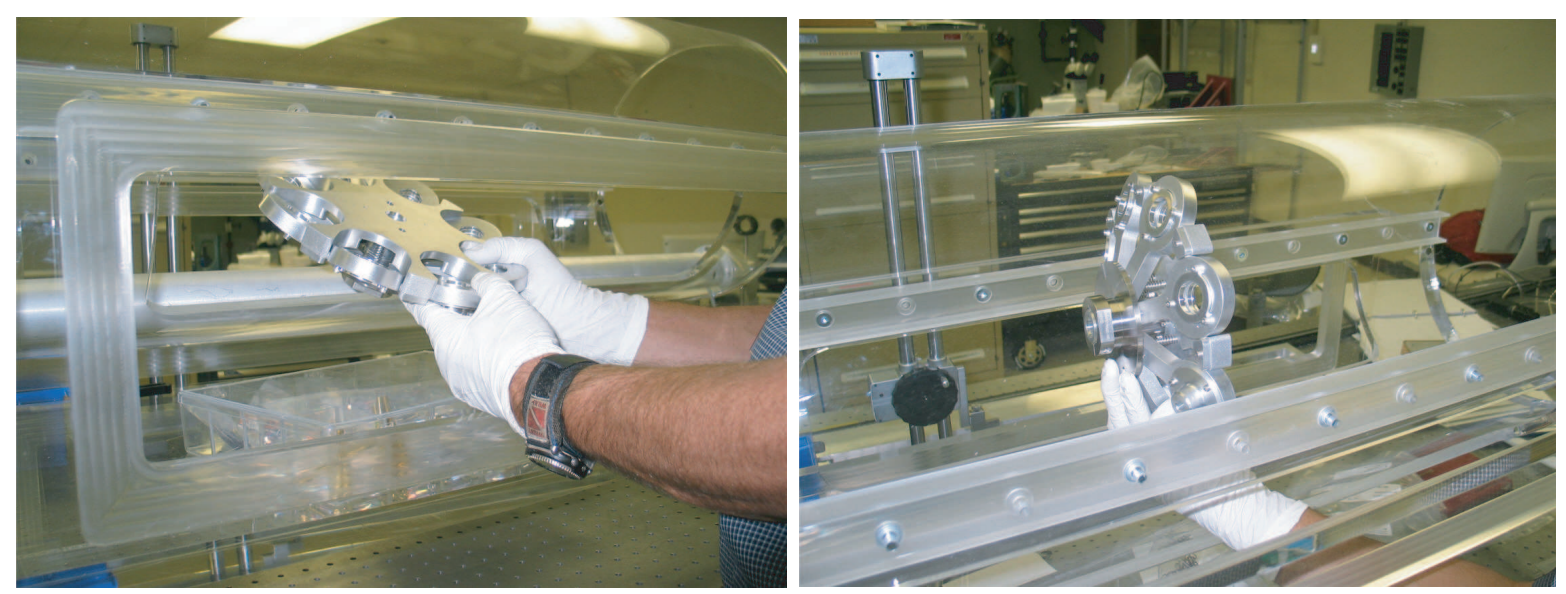

FIG. 1. (color online) (left) XRSA cassette shown being inserted through a mock-up of the DIM side-access panel, showing the space-envelope constraints that allow changes of cassettes between NIF shots. (right) The XRSA cassette in the space envelope of the DIM.

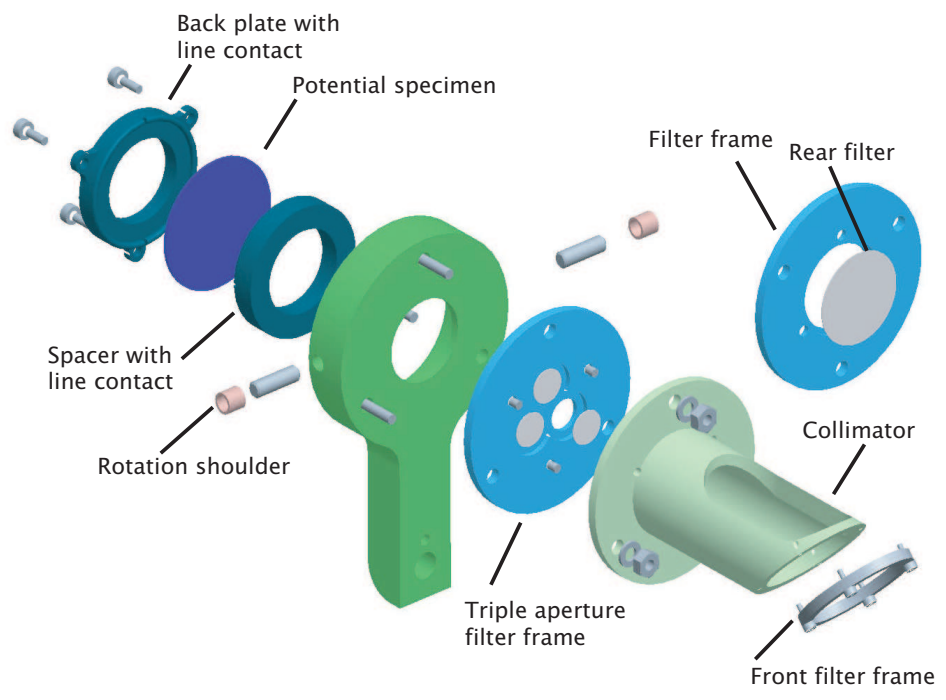

FIG. 2. (color online) Exploded view of one of the eight samples holders or "paddles" in the XRSA cassette. The sample is held in a receptacle in the back side of the green part in the middle of the figure. Filter frames with both single and triple-exposure apertures are shown, although only one type of filter frame was fielded in front of a sample on any shot.

dle), the rotation shoulders that allow the sample's line of sight to be oriented to the x-ray source at the center of the chamber, the filter frames and collimators that held the front and rear filters (discussed further in Section II B) and the sample spacers that isolate the sample from the filters and sample-holder body (discussed in the following paragraph).

We needed to have as little contact as possible between the specimen's front and back surfaces and the sample holder. This is so that in future tests we will be able to monitor the response-induced bending of the sample created by thermal line moments, which are factors of stress gradients generated through the specimen thickness induced by the temperature gradient and changes in material properties that result from the x-ray load. This led us to machine a thin "line contact" into the sample spacer parts shown in Fig. 2 that had contact with the front and back surfaces of the test specimens; the front-side spacer with the line contact is seen as the dark-blue part in the receptacle in the assembled sample holder in Fig. 3. The sample would then be inserted into the receptacle onto the spacer. The point of this minimal contact was to allow the specimen the maximum amount of flex in response to x-ray loading. In the future, we want to be able to measure ${ }^{10}$ back surface motion of the specimens, and large contact surfaces between a specimen and the sample holder would impede that motion. Such a back-surface-motion measurement is also the reason the back plate that confines the sample (the darkblue piece at the back of the exploded view in Fig. 2) is open in the middle. Future designs will also want to minimize stress enhancements in the radial direction by 


\begin{tabular}{|c|c|c|}
\hline Description & $\begin{array}{c}\text { dimension } \\
(\mathrm{mm}, \mathrm{kg} \text { or deg.) }\end{array}$ & comment \\
\hline \multicolumn{3}{|c|}{ cassette dimensions } \\
\hline cassette outer diameter & 256.33 & maximum paddle-to-paddle distance \\
\hline mass of central cassette plate $(\mathrm{kg})$ & 4.5 & \\
\hline mass of assembled cassette and collimators $(\mathrm{kg})$ & 5.0 & \\
\hline \multicolumn{3}{|c|}{ sample receptacle or "paddle" dimensions } \\
\hline paddle outer diameter & $63 \pm 0.3$ & \\
\hline sample receptacle-well diameter (round) & $40 \pm 0.15$ & \\
\hline sample receptacle-well side length (square) & $38.227 \pm 0.127$ & \\
\hline sample exposure-aperture diameter (round) & $25.4 \pm 0.3$ & \\
\hline sample exposure-aperture diameter (triple) & $9.5 \pm 0.15$ & \\
\hline sample exposure-aperture dimension (square) & $25.4 \pm 0.1$ & \\
\hline sample receptacle-well depth (round or square) & $11.5 \pm 0.15$ & \\
\hline \multicolumn{3}{|c|}{ collimator dimensions } \\
\hline collimator upper edge length (single round) & $78 \pm 0.15$ & \\
\hline collimator lower edge length (single round) & $50.93 \pm 0.15$ & $51.13 \mathrm{~mm}$ for triple-round \\
\hline collimator front-edge tilt (degrees) & $45 \pm 1$ & \\
\hline major and minor diameters of elliptical vent hole & $52 \pm 1 \times 24 \pm 0.5$ & \\
\hline \multicolumn{3}{|c|}{ cassette-assembly and mounting rails dimensions } \\
\hline length of rails with attached cassette & 595.67 & referenced to the front mounting nut \\
\hline total mass of cassette and rails $(\mathrm{kg})$ & 9.5 & \\
\hline
\end{tabular}

TABLE I. Table of important dimensions for components of the XRSA cassette. Masses are determined without samples in the receptacles.

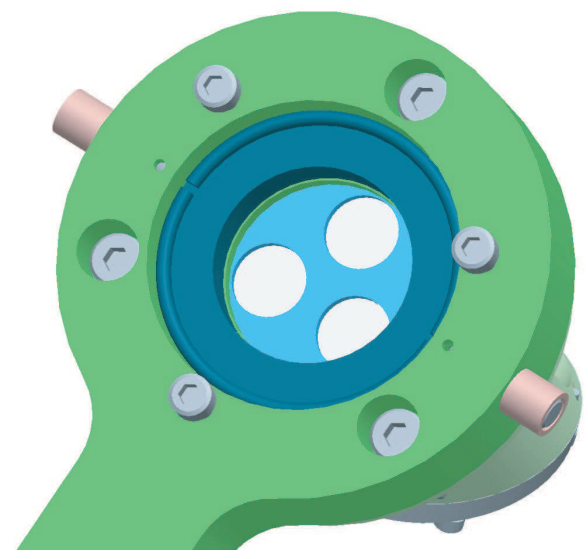

FIG. 3. (color online) Rear view of the spacer with the 'line contact' that separates the sample from the filters and that keeps the sample in minimal contact with the sample-holder structure. The diameter of the round end of the paddle is $63 \mathrm{~mm}$, the diameter of the sample receptacle is $40 \mathrm{~mm}$.

(potentially) bonding the specimen around the edge to the holder with a soft elastomer.

As part of our first test series, we had to be able to accommodate square samples that would be no bigger than $1.5 "$ on an edge; sample-holder paddles with square receptacles and square apertures were also made for this campaign, see Fig. 4. The square samples that were tested had solder contacts that were within the open area of the square-paddle aperture, and that we wanted to protect from direct $\mathrm{x}$-ray exposure. Hence, the spacer plate shown in Fig. 4 had an aperture slightly smaller than the exposure aperture in the paddle, which meant it covered the solder contacts in the manner seen in the right image in Fig. 4.

In order to resolve the damage level within a reasonable uncertainty for each sample, we needed to have up to three noninteracting regions for filtration on each test specimen. One can see two designs for filter frames that go over the aperture in front of the round sample holder in Fig. 2. The designs provides triple and single exposure areas for round specimens with the two filter plates (lightblue items) in the exploded view in Fig. 2. The assembled view in Fig. 3 is set up for a triple exposure. A drawing of an assembled cassette with square and round apertures, with multiple single and triple-filter frames is shown in Fig. 5 .

We needed to provide clocking or indexing of the specimens (and filters) in order to have unambiguous postshot analysis of exposed regions on specimens. This is accomplished by machining reference numbers into the cassette body at each sample position and into the filter frames at each filter location. We marked the edge of each specimen with a small position mark relative to one of the indices in the filter frame. This scheme requires careful assembly of the sample-holder paddle and placement of the specimen according to a systematic, documented protocol that confirmed the orientation and filter mate- 

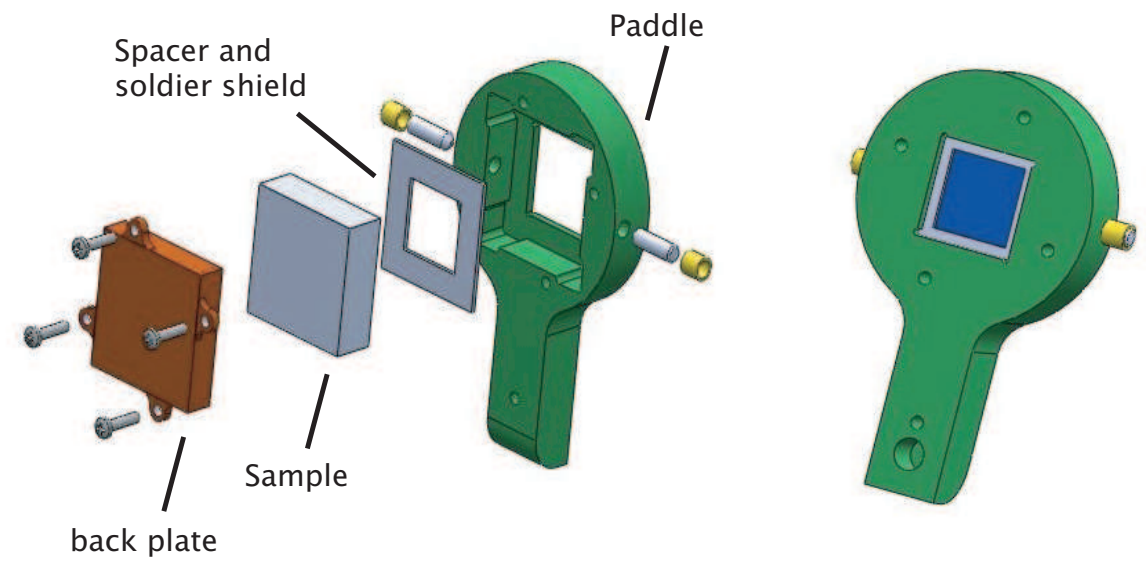

FIG. 4. (color online) (left) Exploded view of a square sample holder and (right) front view of an assembled paddle with the sample surface and the inner edge of the spacer visible in the square aperture.

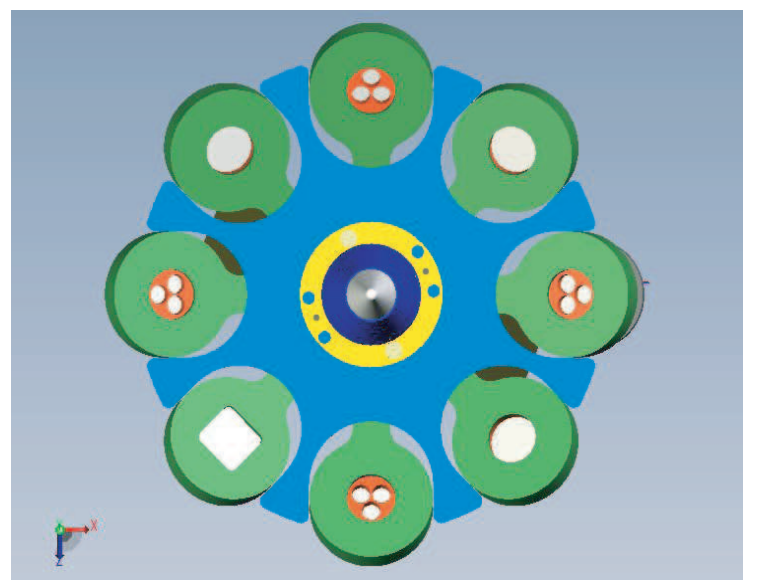

FIG. 5. (color online) Layout of an assembled cassette with sample positions having the various required apertures.

rials at each location. An example of the assembled cassette before the addition of filter frames and collimators is shown in Fig. 6.

\section{B. Filter Pairs and Collimator Design}

For the present experiments, we needed to craft the spectral energy content of the x-ray fluence onto the samples. This is accomplished by mounting paris of filter films in front of the sample surfaces. Typically, we would use an $8 \mu \mathrm{m}$-thick polypropylene film as a sacrificial frontfilter, and a $25 \mu \mathrm{m}$-thick Kapton (polyimide) film as the main (rear) filter. The front filters were located at the end of collimator fixtures, and the rear filters were contained between the filter frame and the collimator base, as shown in the exploded view in Fig. 2. This combination of front and rear filter materials removed unwanted sub-keV x-rays from the radiation incident on the sample, and transmitted a mostly unattenuated multi-keV spectrum $^{3}$.

We had a complimentary requirement for our design to protect the surface of the samples from interactions with target-chamber debris and to keep vaporized or ablated material from the front filter from interacting with the sample. For the former consideration, the combination of the front and rear filters was adequate to protect the sample. For the latter consideration, we had to accommodate the fact that strong $\mathrm{x}$-ray loading of the front filter material would cause it to partially vaporize, and the remaining material would jet backwards (away from the x-ray source). Thus, we developed a collimator design that provides (i) spatial separation between front and rear filters, and (ii) an escape path for front-filter material that is kicked away from the x-ray source by (partial) vaporization of front filter material. In order to avoid having the front filter remains jet towards the sample, the front filter was tilted at $45^{\circ}$ with respect to the sample-x-ray-source axis. Further, the front and rear filters were separated by a minimum of 2" in the collimator assembly (Table I). In order to allow the remains of the front filters to leave the system after the x-ray exposure, an exit is cut into the upper, or exterior, surface of the collimator, which allows the unvaporized material to move out of the collimator without interacting with the rear filters. An assembled cassette with eight collimators is shown in Fig. 7, where one can see collimators of round and square shape bolted to the front of the XRSA sample cassette.

Design calculations show that the ablatively driven front filter material moves away from its initial position with a velocity $\approx 0.5 \mathrm{~km} / \mathrm{s}$ along the normal to the film's surface. Thus, the front filter film transits out of the 1" collimator inner bore in $\approx 70-100 \mu \mathrm{s}$. Additional simulations indicate that characteristic velocities of target material in the NIF chamber are on the order of $150 \mathrm{~km} / \mathrm{s}$, which means that target debris reaches the position of the front filter in the NIF chamber (a distance of $\approx 36-50 \mathrm{~cm}$ ) in $2-3 \mu \mathrm{s}$. Thus, the front filter material is still present 

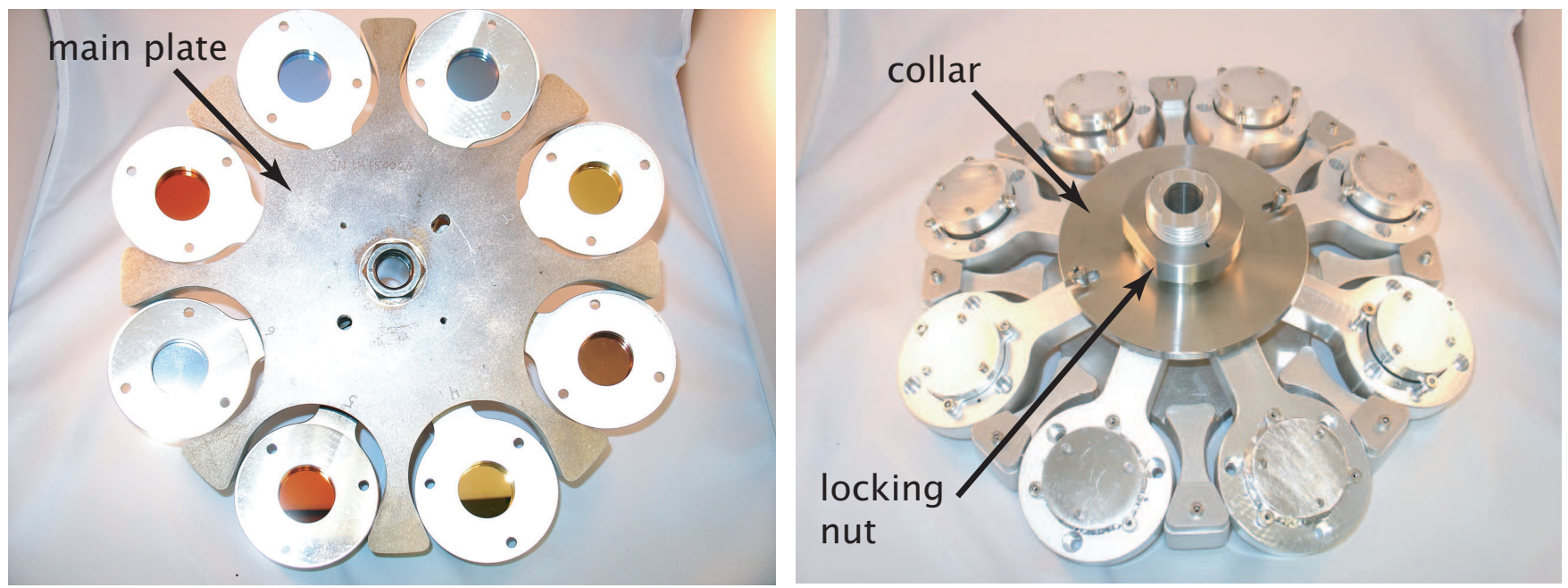

N091110-001-999, SN 14150020

FIG. 6. (color online) (left) Front and (right) rear views of the XRSA cassette, populated with samples and ready for the filter frames and collimators (Sec. II B) to be attached.

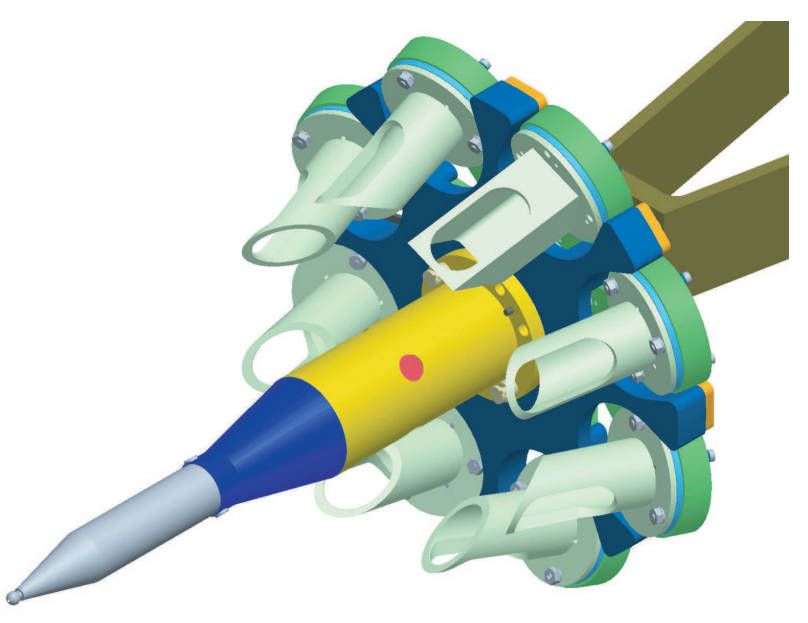

FIG. 7. (color online) Drawing of an assembled XRSA cassette with filter frames and collimators attached. The cassette is is mounted on the rails that connect the cassette to the DIM cart. Also shown is the removable pointer that is used to position the assembly before a shot (see Sec. IID).

in the collimator opening when any target debris reaches that position. Post-shot inspection of over 60 rear-filter films found no target debris on any of them. On about $10 \%$ of the rear-filter films, we found bits of condensed front-filter polypropylene material. There were no penetrations or tears of any of the Kapton films. Thus, we conclude that this tilted two filter configuration provides a large margin of safety for sample surfaces against unwanted interactions with target-chamber debris.

We desired to maximize the data return from each NIF shot, and, for each shot, to provide a resolution of the damage level for each specimen with reasonable uncertainty of at least $\pm 20 \%$. Thus, uncertainties in pre-shot

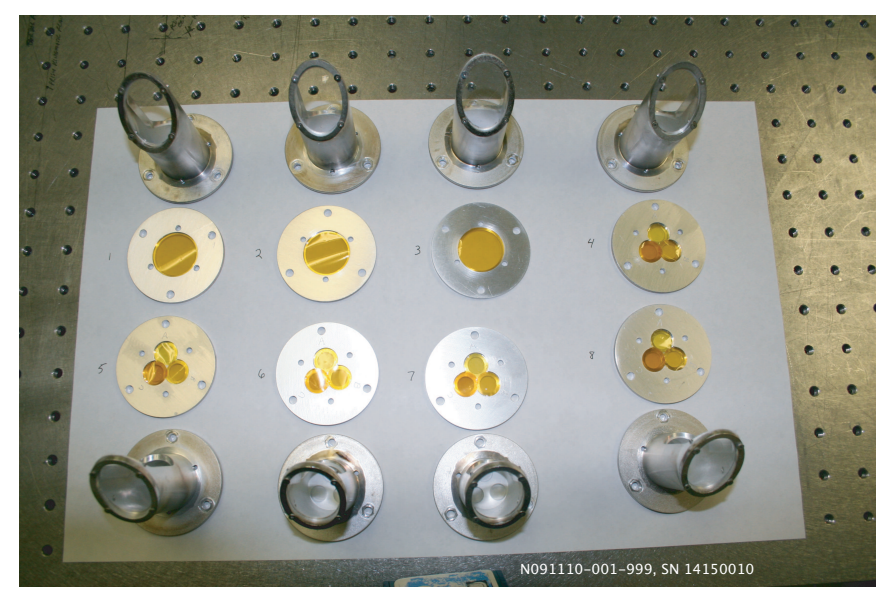

FIG. 8. (color online) Filter materials installed in filter frames and ready for assembly onto the face of the XRSA cassette.

predictions for source output and sample response can be accommodated by having different filtration, e.g. say $12.5,25$ and $50 \mu \mathrm{m}$ of Kapton in the rear filter position, in the three positions of our triple-aperture sample holders. The pre-assembly front and rear filter materials and collimator components for an eight-sample cassette are shown in in Fig. 8. One can see the increasing thicknesses of Kapton in the triple-aperture filter rings by the increasing darkness of the orange color. A completely assembled XRSA test cassette with collimators in front of the sample positions, and front-filter films held taut at the front ends of the collimators is shown in Fig. 9. 


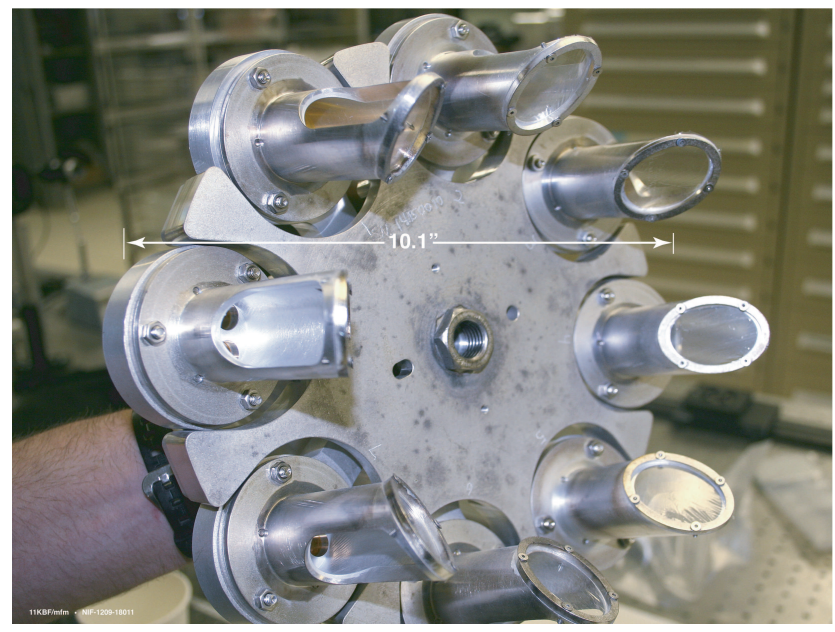

FIG. 9. (color online) Assembled and aligned (Sec. IIC) XRSA cassettes ready for mounting on the DIM rails.

\section{Sample Alignment}

In order to have as uniform irradiation as possible over the samples' surfaces (or to present as large a cross section as possible to a target at TCC), we designed the sample holders to be positioned to look radially at TCC for any stand-off distance. This is accomplished by the rotation pins that couple each paddle to the main plate of the cassette. The bottom end of each paddle is then held against a round, adjustable collar at the back of the cassette (see the right image in Fig. 6) by the compression provided by a spring mounted in the rear face of the main (front) plate of the cassette. The alignment of the samples is accomplished as described below; once the alignment is set, the tilt of the paddles is locked in with the locking nut seen on top of the collar seen in Fig. 6. The XRSA cassette can provide samples with a line of sight to TCC for sample-to-TCC stand-off distances between 320 and $3000 \mathrm{~mm}$.

To begin the sample alignment procedure, the XRSA cassette is installed on the extension arm support stud with which it will be loaded into the DIM, see ' $A$ ' in Fig. 10. This support stud has bosses which mate with recess in the sample plate to ensure that the cassette is clocked in the correct orientation. The outer edges of the paddles are rotated approximately $30^{\circ}$ from the vertical axis of the target chamber; this assures that the XRSA cassette will have maximum clearance from the NIF laser beams. The captive nut at the front of the cassette is then tightened, locking the cassette into position. A laser pointer is then installed into one of the round sample holders, see 'B' Fig. 10. We then install a mechanical pointer onto the front of the cassette. The collar on the back of the XRSA cassette (also shown in Fig. 6) is then adjusted until the laser beam is pointed at the center of the tooling ball on the end of the pointer (see image ' $\mathrm{C}$ ' in Fig. 10). The screw on the back of the collar is then tightened to lock the alignment in place. For thoroughness, the laser pointer is installed in multiple sample locations to verify all sample holders are adjusted equally. When a sample-to-source stand-off distance is required other than one for which a specific mechanical pointer exists, we follow a similar procedure using a surrogate TCC reference (a frosted glass plate with cross-hairs etched onto it). The surrogate is set at the prescribed distance from the front face of the cassette, and its $\mathrm{X}$ and $\mathrm{Y}$ position is verified by the reference K\&E (Keuffel \& Esser) 71-2022 Autocollimating Alignment Telescope that determines the alignment-station centerline. Then, all eight sample positions are adjusted until the laser pointer points to the surrogate TCC reference.

\section{Cassette Alignment}

In order to operate the XRSA cassette safely in the NIF target chamber, we had to avoid interfering with other diagnostics present, the target positioning (TARPOS) system hardware, and to avoid the envelope of unconverted $1 \omega$ laser light that propagates in the chamber with the $3 \omega$ UV light. It is this latter constraint that limits our $256.33 \mathrm{~mm}$-diameter XRSA cassette from having specimens at locations closer than $30.3 \mathrm{~cm}$ to target chamber center (TCC) for the laser configuration described in Fournier et al. ${ }^{3}$. At that distance, in order to satisfy the facility's safety requirements, we needed to know the insertion depth of the cassette relative to TCC to $\pm 0.5 \mathrm{~mm}$ in $\mathrm{Z}$ (radial direction), and the angular orientation of the sample-holder paddles with respect to the target chamber's vertical axis to $\pm 3^{\circ}$. In order to have precise positioning of the samples in the target chamber so that they would receive the expected x-ray fluence, and in order to satisfy the safe-operating constraints above, we used a mechanical pointer and the NIF's Opposed Port Alignment System (OPAS, see Appendix A) to register the XRSA cassettes at a know position relative to TCC. An example of a detachable pointer is shown mounted on the XRSA cassette in Fig. 7.

The alignment procedure for the XRSA cassette in the NIF chamber is as follows: The XRSA cassette is attached to the extension rails that serve as the interface for mounting in the DIM cart. The cassette-and-rails assembly, or DIM load package (DLP), is then inserted through a rear hatch into the DIM housing. After the proper vacuum level is achieved the DIM cart with DLP is inserted into the target chamber. The $\mathrm{X}-\mathrm{Y}$ ( $\mathrm{Z}$ being the insertion-depth dimension) orientation of the DLP is set using the OPAS camera opposite the DIM being used. A circle centered at $(0,0)$ in $(X, Y)$ is drawn on the operator's image at the known size (for the given magnification of the OPAS view) of the front mounting nut that connects the cassette to the rails (Fig. 11). The DIM's bipods are then adjusted so that the image of the nut is concentric with the reticule circle. The DLP is retracted, the DIM is vented and the mechanical pointer is then attached to the XRSA cassette. The DIM is again pumped 


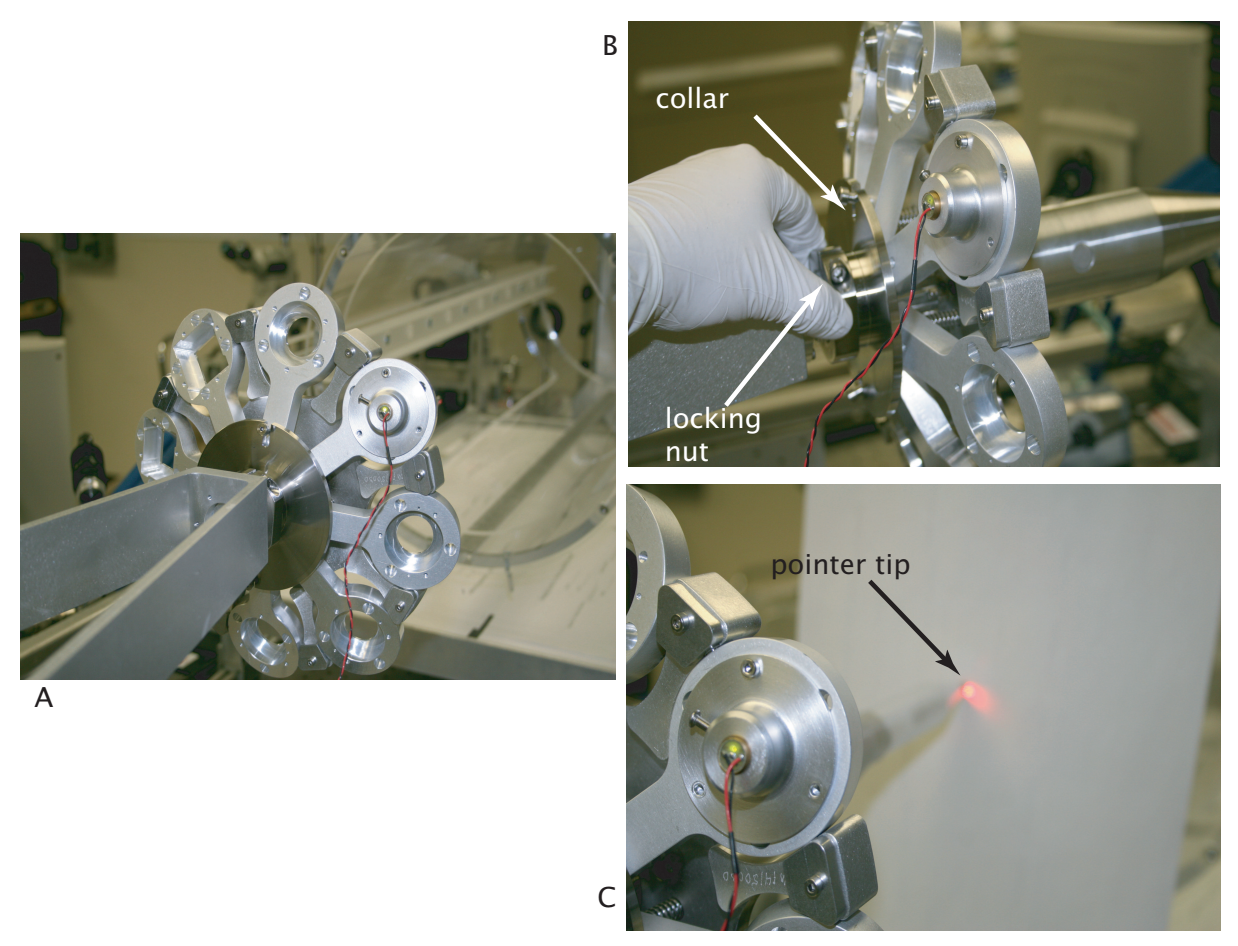

FIG. 10. (color online) (A) XRSA cassette with laser pointer attached to extension arms, (B) adjustment of the collar on the back of the cassette to change the tilt of the paddles, and $(\mathrm{C})$ laser beam aligned to the tooling ball on the end of the pointer.

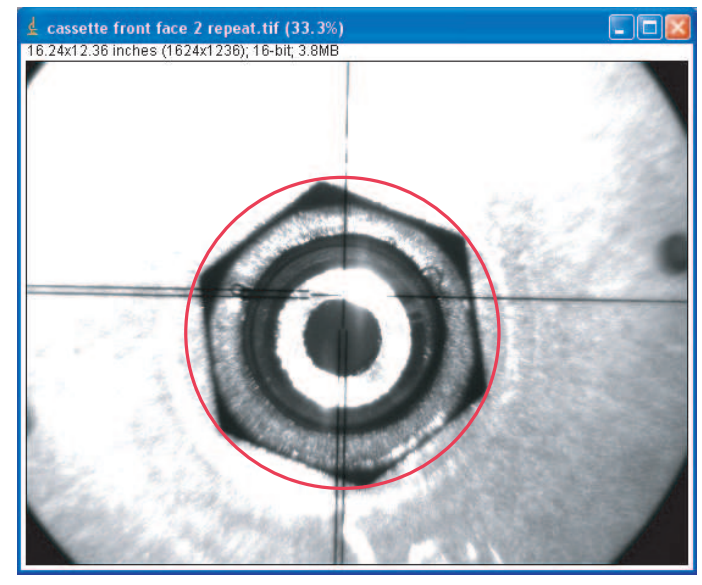

FIG. 11. (color online) OPAS image of XRSA cassette mounting nut, which measures $27.22 \mathrm{~mm}$ corner to corner. The circle reticule is set on the operator's screen at $(\mathrm{X}, \mathrm{Y})=(0,0)$. The DIM alignment is then moved until the nut's image best matches the circle template.

and the DIM cart is inserted into the target chamber and driven to the location that places the spherical ball at the tip of the XRSA pointer at TCC. The location of the pointer tip at TCC is verified by direct imaging with two orthogonal views with the OPAS cameras, which provide an accuracy at TCC to within $\approx 25 \mu \mathrm{m}$. An image is then recorded with the Chamber Interior Viewing System (CIVS, see Appendix B) fixed-view camera, see Fig. 12. The pixel location of a fiducial point, any point with good contrast, usually the left-hand-side inside corner of the extension arms, is noted. The DLP is then retracted to the position that places the samples at the desired stand-off distance from TCC. Again, a CIVS image is recorded and the pixel location of the fiducial point is noted. The DLP is retracted from the chamber and the pointer is removed from the XRSA cassette. The DIM is again pumped down and the DLP is inserted to the saved coordinates for the desired stand-off distance. The location of the fiducial point is again verified in a CIVS image. During operation, it was seen that the XRSA cassette could return to the desired position to within the spatial resolution of a CIVS pixel element, $\approx 0.6 \mathrm{~mm}$.

\section{OPERATION AND RESULTS}

A completely assembled XRSA test cassette with collimators in front of the sample positions, and front-filter films held taut at the front end of the collimator is shown in Fig. 9. The cassette has had the eight sample positions aligned (Sec. II C) to look at the x-ray source located at the center of the NIF target chamber. Two XRSA cassettes are shown in their aligned positions (Sec. II D) in the NIF target chamber moments before a shot in Fig. 12. 


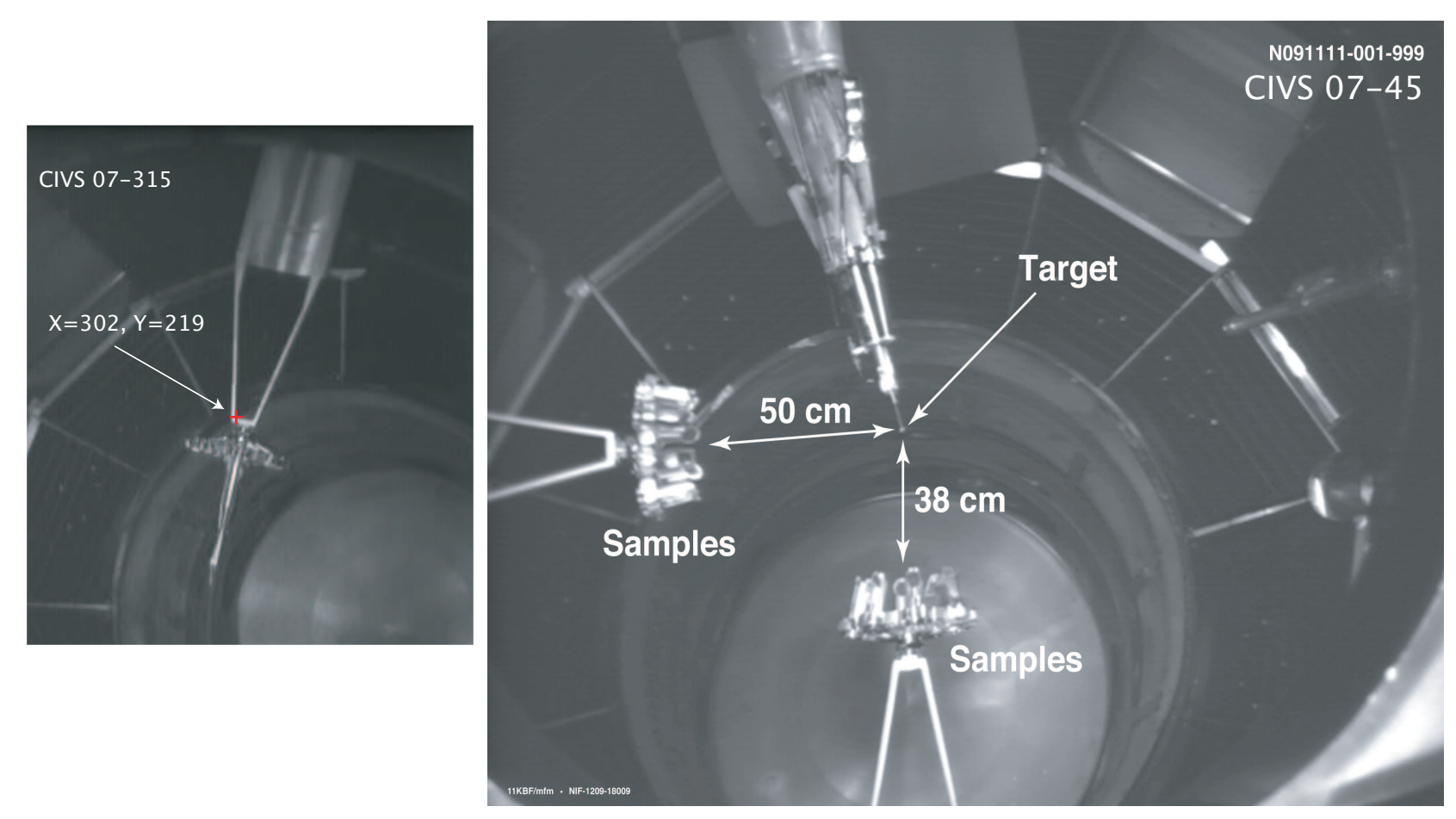

FIG. 12. (color online) (left) CIVS reference image taken with the Polar CIVS wide-field camera during XRSA-cassette alignment procedures. (right) Chamber view of XRSA cassettes aligned moments before a shot. The samples are in DIMS at $(\theta, \phi)=(90,45)$ [bottom] and $(90,315)$ [left], and the TARPOS with the laser target enters the chamber from a port at $(90,239)$ [top].

\begin{tabular}{lcc}
\hline \hline Shot number & $\begin{array}{c}\text { DIM 90-45 SOD DIM 90-315 SOD } \\
(\mathrm{mm})\end{array}$ & $\begin{array}{c}(\mathrm{mm}) \\
\text { N091012-003-999 }\end{array} 340$ \\
N091103-001-999 & 450 & \\
N091104-002-999 & 340 & 500 \\
N091110-001-999 & 450 & 400 \\
N091111-001-999 & 360 & \\
\hline \hline
\end{tabular}

TABLE II. NIF shot numbers and the sample-to-source standoff distances (SODs) for XRSA cassettes operated in NIF's two equatorial DIMs located at target-chamber coordinates $(\theta, \phi)(90,45)$ and $(90,315)$.

\section{A. Filter Film Debris Analysis}

One of the questions to be answered about the operation of the XRSA cassette is how well does it protect the surface of samples held in each receptacle. To that end, we recovered the rear filter film from every sample position from a series of six cassettes exposed to the NIF target environment. The shot numbers and sample-tosource stand-off distances for the six cassettes are listed in Table II. Following inspection both by eye and with a microscope, and photographic documentation, we report that no films were found to have any pinhole punctures or tears. A few ( $10 \%$ or six out of 60$)$ of the rear filter films were seen to have small amounts of particulate debris plated on the target-chamber side of the film (the side opposite to the sample). An example of a 2 mil thick Kapton film recovered from NIF shot N091104-002-999 is shown in the left image in Fig. 13, the dotted circle drawn on image represents the 1" aperture that let $\mathrm{x}$ rays pass through to the sample area. The right hand images are photo micrographs at magnifications of $50 \times$ and $100 \times$ that focus in on one of the regions of particulates. Based on inspection, we determined that these particles of molten polypropylene from the front filter that impacted the Kapton. Based on approximately 60 films that were recovered, we can state that $0.5,1$ and 2 mil thick Kapton films were adequate debris protection for samples (or debris collection surfaces) during this type of target shot. (As an aside, the rear filter also served as a "catcher" of the sample blow-off from the samples under test, and this debris is being used for postmortem test-sample analysis.)

\section{B. Sample Exposures}

A photo of a XRSA cassette following NIF shot N091111-001-999 is shown in Fig. 14. The collimators and rear filters have been removed, allowing one to see the post-exposure sample surfaces. It is possible to see through some of the round apertures areas on some of sample surfaces where the incident x-radiation has caused 

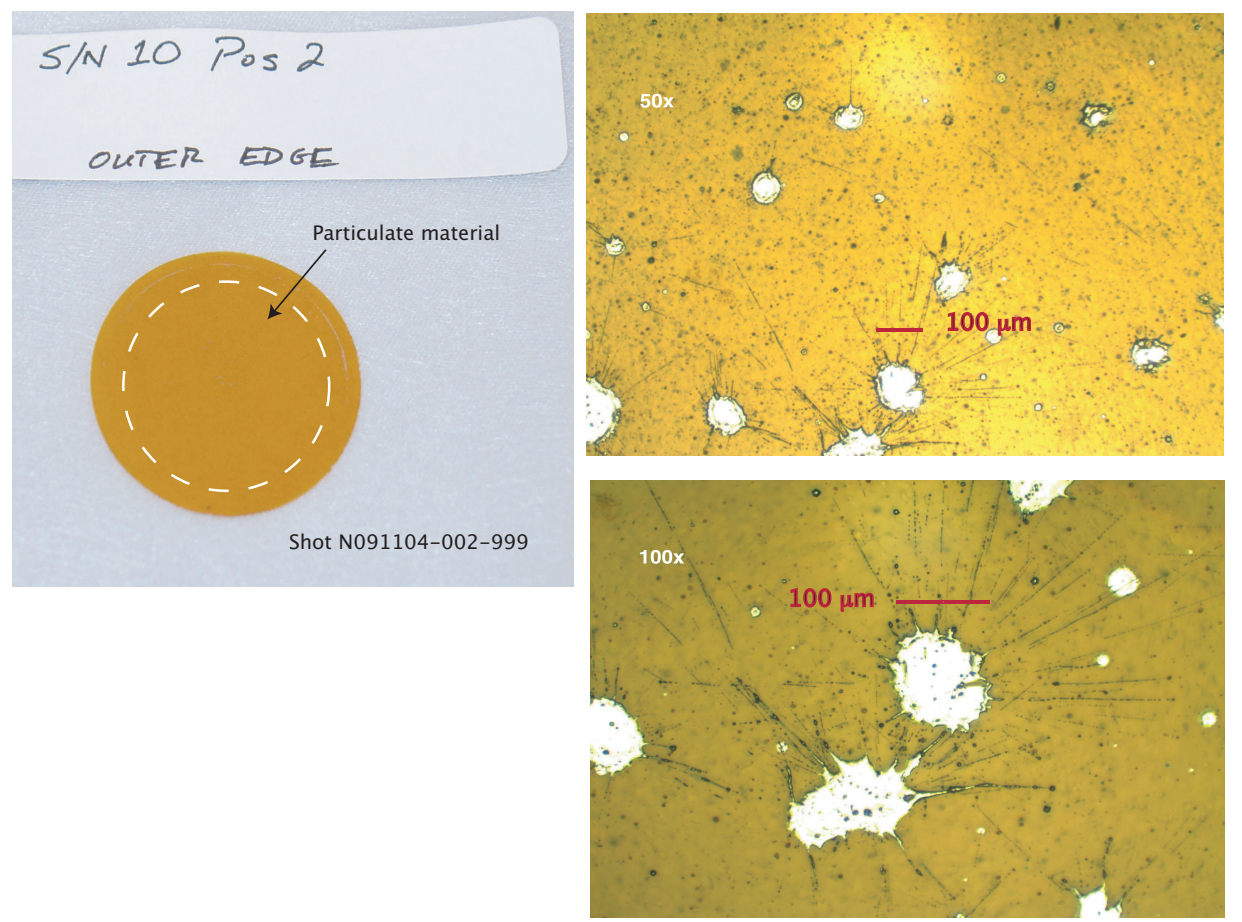

FIG. 13. (Color online) (left) A photograph of a $50 \mu$ m-thick Kapton film used as a rear filter in the XRSA cassette, and (right, top) a photomicrograph at $50 \times$ and (bottom) $100 \times$ magnification of some of the observed particulate debris.

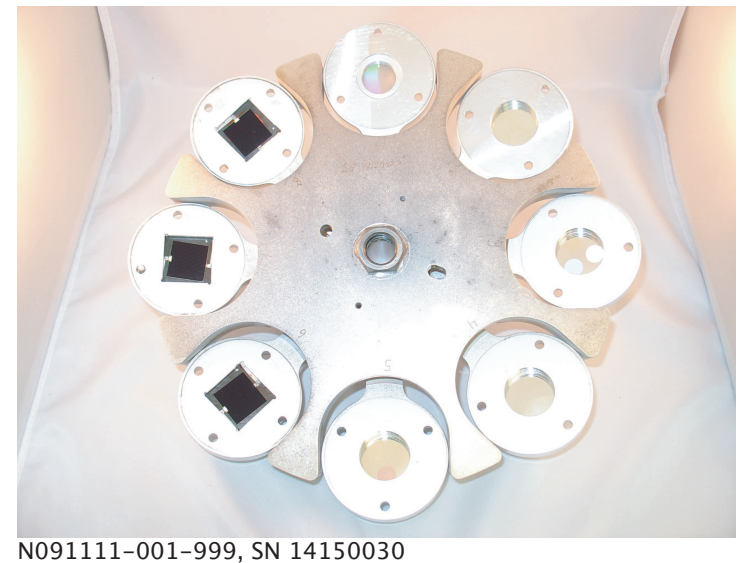

FIG. 14. (color online) Post-shot view of samples in the cassette showing the square and round samples. For this shot, the round sample positions had a mixture of single and triple exposure regions for various samples.

changes in the sample characteristics. Before and after images from one of the samples exposed on shot N091111001-999 are shown in Fig. 15. One can see that the pre-shot surface (left) is featureless and extremely clean. The post-shot surface has regions where x-rays coming through the holes in the triple-aperture plate have changed surface properties. As is mentioned in Sec. II A, the samples are placed in the holder with a fiducial mark to register their orientations. Additionally, as stated in
Sec. II B, the triple-aperture sample holders accommodate a range of rear-filter thicknesses; the filter assemblies are bolted to the face of the sample holder such that the thinnest rear-filter material is at the outermost position in the sample holder with respect to the cassette axis. In Fig. 15, this would be the exposure area at the bottom of the picture. Our convention is that the filters go in order of increasing thickness in a counter-clockwise direction. Hence, the exposed region above and to the left of the lower region has had the next thickest filter material in front. Finally, the thickest filter stack was in front of a position on the sample surface above and to the right of the bottom exposure. No visible change in the surface properties can be seen at this position.

\section{SUMMARY AND CONCLUSIONS}

We have presented the design and operation of a cassette that can position eight samples in the NIF target chamber for experiments during target shots. The cassette provides options for square and round samples and exposure areas; the cassette provides for multiple levels of filtration on a single sample, which allows dynamic range in experiments. The cassette is designed to provide an orientation for samples that is aligned directly to the radiation source at TCC. Using mechanical pointers and facility imaging capability, the XRSA cassette can be positioned at a known sample-to-source stand-off distance with an accuracy of better than a few hundred 

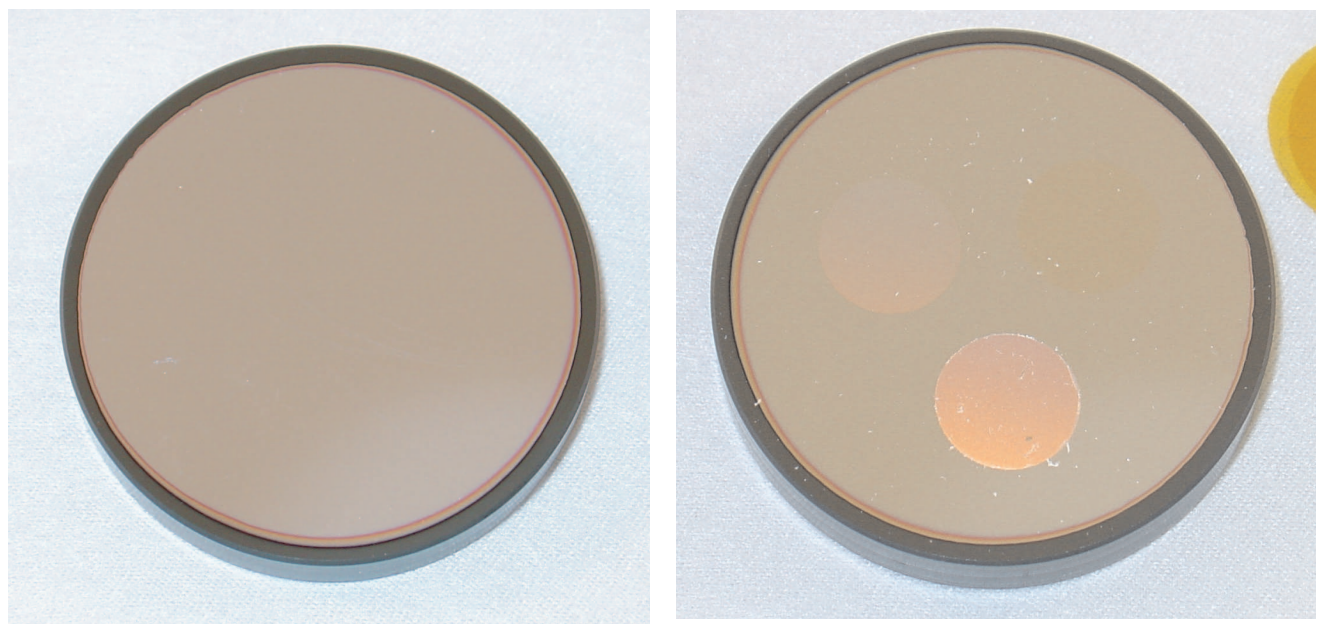

FIG. 15. (color online) (left) Pre-shot picture of a test specimen and (right) post-shot exposed sample from shot N091111-001999, (cassette serial number 14150030 in DIM 90-45, position 3), which was positioned at a sample-to-TCC stand-off distance of $38 \mathrm{~cm}$.

microns. This test cassette has been shown to be useful for experiments that study x-ray interaction with matter. This test-cassette design may also be useful for collecting radiochemical samples following ignition experiments at NIF, or, for providing collection surfaces with which target-chamber debris can be characterized.

\section{ACKNOWLEDGMENTS}

The authors are grateful to the whole NIF team for outstanding support during these experiments. The contributions of working group leaders John Edwards, David Eder, Sandra Brereton and Dan Kalantar were particularly useful for refining the design of our test cassette, Aaron Fisher and Nathan Masters gave excellent support with simulations and analysis. Also, within the Engineering Group, Jared Ellefson and Tony Lee provided valuable discussions and assistance. This work performed under the auspices of the U. S. Department of Energy by Lawrence Livermore National Laboratory under Contract DE-AC52-07NA27344. This work was also supported by the Defense Threat Reduction Agency under the IACRO 09-45501 "Evaluation of Lasers for XRay Production on NIF".

${ }^{1}$ E. I. Moses, R. E. Bonanno, C. A. Haynam, R. L. Kauffman, B. J. MacGowan, and R. W. Patterson Jr, Eur. Phys. J. D 44, 215 (2007).

${ }^{2}$ C. A. Haynam, P. J. Wegner, J. M. Auerbach, M. W. Bowers, S. N. Dixit, G. V. Erbert, G. M. Heestand, M. A. Henesian, M. R. Hermann, K. S. Jancaitis, et al., Appl. Optics 46, 3276 (2007), ISSN 0003-6935.

${ }^{3}$ K. B. Fournier, M. J. May, J. D. Colvin, et al., Multi-keV XRay Source Development Experiments on the National Ignition Facility, (2010), submitted to Phys. Plasmas.

${ }^{4}$ K. Fournier, C. Sorce, M. May, M. Tobin, V. Rekow, D. Beutler, C. Coverdale, R. Gallegos, K. Dudley, D. Osborne, et al., J. Radiat. Eff. 26, 99 (2009).
${ }^{5}$ J. Fisher, C. Newlander, K. Fournier, M. May, M. Tobin, D. Beutler, C. Coverdale, J. Davis, and D. Shiekh, J. Radiat. Eff. 26, 59 (2009).

${ }^{6}$ K. B. Fournier, J. H. Fisher, D. Osborne, T. Zarick, M. J. May, J. D. Colvin, C. Sorce, J. Emig, V. Rekow, C. D. Newlander, et al., J. Radiat. Eff. 28, 33 (2010).

${ }^{7}$ G. P. Grim, P. A. Bradley, T. A. Bredeweg, A. L. Keksis, M. M. Fowler, A. C. Hayes, G. Jungman, A. W. Obst, R. S. Rundberg, D. J. Vieira, et al., Rev. Sci. Instrum. 79, $10 \mathrm{E} 503$ (pages 5) (2008).

${ }^{8}$ P. Bell, D. Lee, A. Wootton, B. Mascio, J. Kimbrough, N. Sewall, W. Hibbard, P. Dohoney, M. Landon, G. Christianson, et al., Rev. Sci. Instru. 72, 492 (2001).

${ }^{9}$ W. J. Hibbard, M. D. Landon, M. D. Vergino, F. D. Lee, and J. A. Chael, Rev. Sci. Instru. 72, 530 (2001).

${ }^{10}$ D. H. Dolan and S. C. Jones, Rev. Sci. Instru. 78, 076102 (2007).

\section{Appendix A: OPAS}

The OPAS is a modified reflecting telescope line of sight (LOS) aligned to be co-linear with the DIM axes, i.e., one at 90-225 opposite DIM 90-45 and one at 90-315 opposite DIM 90-315. Each OPAS LOS has two cameras that provide narrow and wide fields of view. The cameras sit on a station outside the target chamber. The system uses a motorized stage for the collector plate and secondary mirror that allows focus adjustment from TCC to the opposite chamber wall. The telescope is adjusted so that moving the focus does not walk the image. The field of view of the wide focus camera encompasses a volume with an $8 \mathrm{~cm}$ diameter at TCC. The OPAs provides the capability to align a DIM-based diagnostic with respect to TCC, and to align a target near TCC. DIM-diagnostic alignment may be done to an accuracy of $25 \mu \mathrm{m}$ near TCC. 


\section{Appendix B: CIVS}

The CIVS consists of 10 radiation-hardened video cameras (Industrial Video Systems IVS 400 and Sony FCB-EX1000P) located on seven target chamber ports. Three ports have two fixed camera views for calibrated reference images. The wide field of view $(\mathrm{FOV})$ is $\approx 2 \mathrm{~m}$ in diameter, the narrow $\mathrm{FOV}$ is $\approx 40 \mathrm{~cm}$ in diameter. Four ports have adjustable camera views with pan/zoom capability for survey investigations. The CIVS units are intended to provide stable calibrated views for system operators. The fixed magnification (pixel calibration) allows quantitative assessments of distance between positionings. Fixed pointing allows one to reference TCC with a specific pixel location. For each viewing direction, the wide FOV resolution is $\approx 2.5 \mathrm{~mm} /$ pixel, while the narrow FOV resolution is $\approx 0.6 \mathrm{~mm} /$ pixel. 